Voix et Images

voixetimages

\title{
Dessins, autoportraits et autres figurations
}

\section{Gabriel Landry}

Volume 27, numéro 2 (80), hiver 2002

La sociabilité littéraire

URI : https://id.erudit.org/iderudit/290062ar

DOI : https://doi.org/10.7202/290062ar

Aller au sommaire du numéro

Éditeur(s)

Université du Québec à Montréal

ISSN

0318-9201 (imprimé)

1705-933X (numérique)

Découvrir la revue

Citer cet article

Landry, G. (2002). Dessins, autoportraits et autres figurations. Voix et Images, 27(2), 346-353. https://doi.org/10.7202/290062ar d'utilisation que vous pouvez consulter en ligne.

https://apropos.erudit.org/fr/usagers/politique-dutilisation/ 


\section{Dessins, autoportraits et autres figurations}

Gabriel Landry, Collège de Maisonneuve

Par le titre de son plus récent livre ${ }^{1}$ et notamment par l'avantdernière pièce, éponyme, du recueil, Robert Melançon nous rappelle que la réalisation du poème est comme celle du dessin affaire d'esquisses et de ratures, de brouillons, de recommencements, de traits improvisés et de motifs élus. Le dessinateur sousentend également que la figuration 
est au cour du travail poétique, quoi que stipulent certains canons modernes.

Ces idées ne sont pas nouvelles dans la poésie de Melançon. Plusieurs poèmes de Peinture aveugle ${ }^{2}$, polarisés par la métaphore de la page paysage ${ }^{3}$, tenaient lieu d'arts poétiques en comparant les entreprises du peintre et du poète, tous deux s'employant à "retracer les contours ${ }^{4}$ "de la réalité fuyante. Tous deux occupés à des travaux de composition et de retouche engageant le regard. Aussi, pour qui connaît l'œuvre de Melançon poète, cette observation tirée des "Notes d'une poétique négative" acquiert-elle une sorte de plus-value:

La poésie représente toujours quelque chose. Elle est, par nécessité, un art figuratif: les sons, les couleurs ne prennent que les significations qu'on leur prête; mais les mots en ont toujours $\bar{a}$ l'avance, qui donnent au poète son matériau'.

Je ne vois personne d'avisé, fabricant ou lecteur de poèmes, qui songerait à contester ces propos-lá. On peut toutefois leur adjoindre ceci : si toute poésie, à l'instar de tout langage, est en effet forcément figurative, celle de Robert Melançon l'est sans doute plus qu'une autre. En témoignent, dans Le dessinateur, des intitulés éloquents: "Paysage avec une figure", "Description d'un aprèsmidi", "Description d'un jour d'automne", "Pochades en vers", "Retouche", "Paysage minimaliste", "Dessins", et c'est sans compter les références à la photographie et à la peinture (Hokusaï, Cézanne, Georgia O'Keeffe). En témoigne surtout une écriture de la précision et de la mesure qui fait de chaque poème un tableau au rendu et aux contours nets ${ }^{6}$. Sans s'y réduire, le poème de Melançon consent à l'acte de décrire, voire à enregistrer comme sans façon le réel ambiant, parfois le plus ordinaire :

Ce lendemain de l'Halloween, Les rues sont couvertes de feuilles [...] On voit sur les bakcons des citrouilles, Des poupées de jute, des pendus

Taillés dans de vieux draps, que le vent Balance dans ces rues... (p. 27)

On aime mentionner que Melançon est un spécialiste de la poésie de la Renaissance, dont l'héritage était manifeste dans Peinture aveugle? mais le poète du Dessinateur ressemble davantage à un classique. Sa sobriété et son goût des "motifs pauvres" (par exemple un érable dans la nuit d'hiver: "Je prends à contempler ce motif pauvre/Une joie qu'ignorent les dieux ${ }^{8}$ ", p. 43), son refus du grossissement et de l'onirisme, bref, sa relative simplicité et sa lisibilité, le situent à côté non seulement d'une influence proprement baroque ou maniériste, mais encore de quelquesunes des tendances les plus notoires de la modernité. Les traits différentiels de la poésie moderne sont à toutes fins utiles absents du Dessinateur: hermétisme, excentricité, abstraction, autoréflexivité, thématiques du corps et du désir, dictature de l'imaginaire. On pourrait même avancer qu'avec Melançon, le poème descend de son piédestal symbolique et touche au sol, au concret de l'espace environnant, celui de la ville par exemple (Montréal précisément), dont il dessine la "géométrie aérée" (p. 43):

J'ai toujours aimé ce jardin dont la haie de lilas

Exclut cle tous côtés l'horizon. La nuit s'y fait plus dense 
Et la rumeur de la ville tout autour S'y fait entendre pour parfaire sa solitude (p. 15).

Il y aurait beaucoup à dire sur l'aspect narratif de tels poèmes, dont l'expansion offre une belle contrepartie à la retenue des haïkus, autre genre cher à Melançon. Le dessinateur est d'ailleurs, en regard de la question des genres, un assez remarquable recueil, autre aspect qui le distingue des publications actuelles. Qu'il s'agisse de genres canoniques ou non, il y a là une une belle ordonnance de l'hétéroclite: lettres, haïkus, limericks, pochades, hommages, nature morte, description, essai de définition... Cette traversée des formats, du ramassé à l'étendu, est peut-être ce par quoi Le dessinateur échappe significativement aux lois (unité, continuité) qui régissent par ailleurs son écriture, écriture que je qualifie de classique" à défaut d'une meilleure caractérisation. À chaque nouveau poème la forme ancienne s'évanouit dans un rythme neuf. La trame continue de clurée s'abolit par là dans une diversité qui n'est pas sans rappeler le goût baroque de l'inconstance ou de la métamorphose. Ces variations sont peut-être, aussi, l'aveu discret d'un dessein impossible à atteindre une fois pour toutes. Chaque poème du Dessinateur recommence à figurer ce qui se dérobe sans cesse. Parfois même ce qui n'a pas de figure. L'une des plus longues suites du livre, "Air", dont la laisse finale présente un beau mouvement circulaire, réitère par son impossible sujet que le poète a charge de donner à voir l'invisible ${ }^{10}$. Le poète s'acquitte ici de son "devoir" (Melançon n'aimerait probablement pas ce mot, lui qui parle de la poésie comme d'u un jeu/Sans autre objet que d'adoucir le cours du temps ${ }^{11}$ ", p. 26) avec une justesse de ton, une discrétion et une délicatesse qui "sonnent " comme des enseignements.

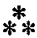

Stéphane Despatie a publié ses premiers livres dans la collection "Poètes de brousse" des Éditions Les Intouchables. Son dernier titre, Garder le feu ${ }^{12}$, appartient encore à l'esprit de cette famille poétique initiale. On y entend une voix qui élève les protestations conjuguées de la révolte et du lyrisme. On y lit des vers qui se veulent proférables, des vers longs imprégnés d'affects et de ferveur missionnaire:

nous gardons le feu en ces temps
qui nous séparent
osons soulever les pierres de la
route de terre
nous polissons la patience à en
faire une lance
comme l'artiste guerrier peint un
soleil sur le ventre
$\quad$ de la nuit (p. 33)

Garder le feu se compose de quelque 140 quatrains de cette mouture-là. Il s'agit en effet de quatrains, le complément du nom "de la nuit" s'avérant un faux rejet, comme tous les autres, simple conséquence d'un manque d'espace pour un vers aussi long. Despatie a composé des quatrains élastiques, les plus étirés du recueil approchant la forme du verset. Il atteint par là à une relative unité rythmique. Et la succession ininterrompue des strophes (ou versets) confère à l'ensemble l'allure d'un seul long poème, prodigue. Cette amplitude convient assez à la 
matière romantique du recueil: la souffrance, le chant amoureux, la fuite des ans, l'avancée vers la mort, la conservation de la mémoire. "[G]ardons la flamme comme l'empreinte de nos origines" (p. 14), dit un vers qui évoque la quête de l'homme primitif aussi bien que le mythe prométhéen. L'écriture de Despatie s'assigne elle-même un rôle de préservation, car elle cherche à revitaliser d'anciennes alliances analogiques: la parole comme flambeau, le vécu comme brûlure, le poème et l'amour comme foyers paradoxaux, sources de chaleur ou propagateurs incendiaires. Mais mis à part ces arêtes vives, la chaîne de quatrains de Garder le feu manque assez de relief. C'est peut-être imputable, étonnamment, à l'aspect très éclaté de la plupart des quatrains. On dirait qu'il leur manque cette unité organique propre aux véritables réseaux de sens. En réalité, les vers et quatrains de Garder le feu charrient des perles et des cailloux, vont un peu dans tous les sens. Il en résulte qu'aucun sens ne semble exploré avec force. Cette impression d'éparpillement tient aussi à une syntaxe dont la complexité paraît souvent superflue. En tout cas, la surabondance des circonstancielles et des compléments du nom annulent ici et là l'effet recherché, le poème se perdant dans une surcharge de couleurs, de figures, de nuances:

dans la chimie des sangs
jusqu'aux lèvres
de l'oeil
les braises sifflent sous les larmes
du brouillard
des patiences (p. 29).

Et que penser d'une séquence comme la suivante?

un accord forcé un rien vêtu de douleur conserve d'autrefois en l'oreille

un chant

d'une charité que j'ignore à

l'espace incalculable

et ce qu'il faut de surdité pour

s'accorder le piano (p. 19)

Dans ce quatrain, à mon avis, le sens ne "prend" pas. La trame accord-oreille-chant-surdité-piano signale une isotopie dont le reste n'a que faire, à moins que l'on soit dans la tête du poète, ce qui est toujours difficile. "IS]acralisant le rêve je masque d'un trait la réalité", lit-on plus loin. Y aurait-il là formulation d'un programme et, partant, livraison d'une clé de lecture? Certes, le poète est libre de masquer ce qu'il veut masquer, sans autre légitimation que son souverain caprice. J'imagine que ce n'est pas chez un éditeur qui s'appelle Planète rebelle qu'on chipote là-dessus. Mais on dirait que l'écriture de Despatie se cherche encore, entre l'abandon à un lyrisme touffu, embrasé, et un "arrangement en poème" plus réfléchi, qui ne craindrait pas d'émonder un peu autour du feu, question de lui donner tout l'air qu'il réclame.

\section{***}

On pourrait penser qu'un intitulé comme La totalité du paysage ${ }^{13}$ annonce un projet hautain ou prétentieux. Il n'en est rien. Le paysage "total", complet, c'est simplement celui qui englobe la mort. Ce thème grave donne lieu, avec Thibault, à des poèmes assez légers, voire comiques. La figure du poète qui apparaît en filigrane de ces soixante-deux poèmes n'est pas celle, romantique, solennelle, du mage ou de l'inspiré. Elle représente un "habitant du 
néant" (p. 16) comme les autres. Pas d'exemption pour le poète donc, et d'une façon plus railleuse:

On naît et puis voilà

tout est dit.

[...]

Holà poète

n'oublie pas que tu mourras

la bouche ouverte! (p. 70)

Les tableaux qui composent le paysage de Martin Thibault découlent d'un regard à la fois amusé et sceptique. Regard sur soi-même autant que sur le monde, qui passe de la sphère du moi à la boule terrestre: "Je tourne en rond/mais j'avance en spirale", dit l'exergue du recueil, à quoi fait écho plus loin

La Terre abrite notre fragilité, sá
force:
tourner en rond sans jannais reve-
nir au même point
et avancer en spirale (p. 64)

Dans certains poèmes, on dirait que ce regard a pris le recul qu'il lui fallait pour rendre des impressions distanciées, qui sont juste assez réservées pour préserver le poème de l'intime ou du sentimental. Juste assez de recul encore pour qu'à l'inversè, cette poésie sollicitée par le cosmos (la totalité du paysage, c'est aussi l'infini interstellaire) n'ambitionne pas de l'embrasser dans un seul poème.

La poésie de Thibault, dans ses meilleurs moments, a quelque chose de leste et de dégagé, ainsi cette "Évidence" (p. 17):

Les idées noires sortent la nuit pour ne pas se faire voir dans leur aveuglement.

Je sais maintenant où je valis Et je vais perdre.

Cet humour, ce constant besoin d'échapper à la gravité (à l'attraction terrestre) ne vont pas sans écueil. Quelques poèmes retombent platement sur terre, se contentant des plus banals constats: "La paix n'a jamais été signée/entre la vie et la mort, les pauvres/et les riches." (p. 36) Là, l'absence de prétention devient carrément de l'indigence. Ces scories n'étaient pas nécessaires; sans elles le paysage n'eût pas été moins "total".

La totalité du paysage ne ressemble pas aux publications courantes du Noroît, maison qui accueille d'ordinaire une poésie plus "essentialiste" ou plus classique, plus sérieuse aussi, ou plus grave, souvent teintée de spiritualité. Comme de raison, que Thibault chante son air à l'écart de l'orchestre ne constitue pas en soi une garantie de qualité, mais ça mérite d'être relevé. Pour la petite histoire de la poésie made in Québec.

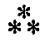

L'écriture de Marc André Brouillette explore des contrées plus austères. Dans ce beau livre, une plaquette de petit format comme on les aime, à la couverture et au titre également marins, Vent devant ${ }^{14}$, une parole s'exerce à l'art périlleux de l'extrême rétention. Cinq sections brèves, qui comptent entre neuf et quatorze micropoèmes: une délicate menuiserie. L'entreprise est d'autant plus audacieuse que Brouillette joue sur un clavier de mots très peu étendu, pas du tout chromatique. Quelques vocables essentiels, stratégiquement distribués, assurent à la partition son harmonie. Ils retiennent, viennent ancrer un imaginaire qui, autrement, échafaude des rêves d'ailleurs et de mouvement. 
Vent devant est un poème de la mer et du voyage, justement. On a tôt fait de saisir la nature allégorique de cette traversée: traversée de soi par l'écriture, ou traversée du poème pour arriver à soi, avec ce que cela implique d'aléatoire, d'intempéries, d'écueils menaçants. Les mots, le langage sont parfois des entraves, ils pèsent de toute leur usure, ce sont des amarres à rompre:

\section{j'aimerais oublier \\ certains mots du passé qui \\ s'imposent \\ et m'arrêtent telle une ancre}

ils retarclent mon départ (p. 67)

En contrepartie,

nait de l'encre

un corps qui

s'ouvre aux sens (p. 43)

Amusante dialectique: arrimée au langage qui la précède, la poésie doit en franchir le seuil, atteindre à ce métalangage qui élargit l'ancien horizon. La poésie est appel d'air, promesse de vent porteur. S'il faut en croire le poète lui-même, dans pareille entreprise il y a loin de la coupe aux lèvres. Le frêle esquif de Vent devant mène en tout cas aux confins du silence. Le texte ensevelit ses métaphores marines sous l'image recouvrante de l'hiver, qui conspire avec les thèmes de l'aveuglement et de la mort: "la parole s'est amuie." (p. 71)

\section{***}

La réduction du verbe au silence n'est pas une hantise pour Maurice Tardif, qui donne avec Autoportraits à la paille creuse ${ }^{15}$ un premier livre très bavard. Rarement recueil fut aussi composite - et aussi inégal!
Un singulier soupir, non celui de la mort mais celui de l'obligation de vivre, parât être le point de départ de l'aventure poétique. Mais vivre et écrire ne se fondent pas toujours en vécrire:

\section{Hélas! je suis toujours debout}

Ardu vivant (p. 25)

"Ardu vivant", comme art du vivant, comme mauvais jeu de mots. Calembours et autres facilités ne manquent pas dans cette masse verbale où le procédé semble une fin plutôt qu'un tremplin. On se lasse vite de pareils chapelets, qui pour un peu s'étireraient la corde jusqu'à la neuvaine:

Ma vie, la percée...
Qui ma vie
Oui ma vie
Par les vents
Les vannes
L'évanescent
Les vents
Les dents
De l'évanouissement (p. 12)

Cette illustration bien plate du phénomène de l'engendrement lexical n'est pas seule de son espèce dans ce recueil où, autre faiblesse criarde, le poème en vers n'est souvent qu'un assemblage mécanique qui étage de la prose. C'est quand il délaisse le vers que Tardif élève une voix qui peut prétendre à la poésie. Dans la meilleure partie du livre, qui s'intitule "Exercices de pointes" mais qui dépasse, elle, l'exercice de thétorique, une prose aphoristique entremêle maximes, définitions, confidences et interrogations. Il y a là-dedans beaucoup de mordant, des raccords trouvés, une jouissance dada. Le tout dans une langue élégante, sans l'emphase qui ruine d'autres moments du livre. Je ne résiste pas au plaisir du plaisir: 
Hier, j'ai vu la mouette, la mouette blanche et nerveuse al long vol étranglé. (p. 81)

J'étais jeune à l'époque. Porter tête ne m'allait pas. (p. 81)

Un revolver rêve d'une balle. Ne le tirez pas de son sommeil. (p. 85)

J'aime cet envoûtement des chenaux de l'esprit qui retourneront à la cascade, à l'arrière-forêt, à la tête encore. (p. 87)

Ma fièvre n'a jamais tombé. Son feu m'a précisé. (p. 92)

De ces Autoportraits, desquels l'auteur dit qu'ils "sont nés [du] néant expressif" de sa "face intacte, rigide, absente" (p. 104), retenons ceux qui dessinent du langage un visage personnel.

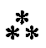

"Je vais prendre un visage pour commencer ": ainsi Normand de Bellefeuille entame-t-il sa dernière déclinaison ${ }^{16}$. Elle est tout de suite aussi prenante que la précédente ${ }^{17}$, qui avait valu à son auteur le Prix du Gouverneur général et celui de l'Académie des lettres du Québec. Un ensemble de douze textes, "Géométrie d'une absente", lui fait d'ailleurs écho en en réactivant le chant litanique, celui de la douleur.

De Bellefeuille est remarquablement fidèle à lui-même, à ses vues anciennes. Il construit depuis trente ans une cuvre qui se renouvelle continuellement. On dirait un cercle dont le centre repousse inlassablement la circonférence, l'extension de la figure ne perdant jamais de vue son noyau central, le régénérant plutôt d'un livre à l'autre. Le tout premier intitulé, "Les choses les cho- $\operatorname{ses}^{18}$ ", comme par hasard projetait à l'orée de l'ouuve la figure fondatrice du redoublement. Trois décennies plus tard, le superbe récitatif d'Un visage pour commencer se ponctue d'une batterie obsessionnelle:

je vais prendre un visage pour commencer

je vais prendre un visage

et le répéter

[...]

prendre les lèvres

ctu visage

les clents et les gencives

les yeux, tout le globe, puis le nez et tous ses habitants je valis prendre ton visage pour commencer (p. 11)

Dans ce dernier livre, la langue de de Bellefeuille ravit aux aimants du désespoir et de l'absence un métal original et précieux. Elle a des antécédents notoires, Whitman, par exemple, ou Lorca, duquel sont cités ici deux vers de "La Blessure et la mort ${ }^{19}$ ", car "[t] out a commencé/par un poème" ( $\mathrm{p} .84$ ). Mais le poète de Bellefeuille n'a pas, comme on dit si bien, "son pareil". Sans trancher des rapports entre cette ouvre et la figuration, on peut dire qu'elle sait mieux qu'une autre produire les effets du prisme dans les images qu'elle suggère d'une beauté aussi fantôme que visible.

1. Robert Melançon, Le dessinateur, Montréal, Éditions du Norôt, 2001. $56 \mathrm{p}$.

2. Premier livre important (substantiel) de Melançon, paru chez VlB en 1979: le poète avaut auparavant publé neuf courts poèmes sous le titre d'Inscriptions (L'Obstdienne. 1978).

3. On retrouve cette métaphore dans Le dessinateur. "La neige viendra, $/ \mathrm{S}_{1}$ égitle que tout/L'espace blanchi/Se recomposera// En tune page neuve. (p 32).

4 Penture aueagle, op. cit., p. 69.

5. "Notes d'une poétique négative ", Poésue of politique, Montréal, l'Hexagone, 2001, 506 p. 
6. Si le poème est un tableau, l'espace de ce tableau a son cadre; on y songe en lisant: "Et du gris traverse/L'espace entoilé. (p. 18). Le verbe entoiler "signifie garnir de toile (sens ici privilégié), mais aussi fixer sur une toile.

7. Notamment, par la récurrence notable de verbes clés (déployer, disperser, dissoudre, verser) exprimant l'écoulement ou la métamorphose.

8. Ce vers rappelle un clistique d'Emily Dickinson, à laquelle $R$. Melançon a consacré de belles pages (dans Liberté, $n^{\circ} 164$, avril 1986): *A Letter is a joy of Earth -/It is denied the Gods - $\rightarrow$ Le rapprochement paraît d'autant légitime que Melançon prarique la lettre comme genre poétique, Le dessinateur s'ouvrant et se fermant sur une lettre au poete George Johnston, textes qui donnent au livre son "adre", ni plus ni moins

9. Classique encore ce goût de la régularité strophique et métrique, même si, dans ce dernier cas, Melancon a souvent recours aux vers que Jacques Réda appelle * mâchés". La régularité dont te parle s'observe à l'échelle du poème, non à celle du recueil.

10. "Hommage à Philip Larkin. (p. 26) désigne semblablement la mort: «ce qui n'lal/Ni forme, ni mesure
11. C'est là une idée toute... malherbienne, le poète n'étant guère plus utile à la société qu'un joueur de quilles. Ces propos extraits des "Notes d'une poétique négative * hygıéniques compte tenu de certains tapageurs, vont dans le mêtme sens: "Un poète porte une cravate, va au bureau, paie ses comptes, fait ses courses, se comporte sous tous rappons comme le premer venu qu'il est *

12 Stéphane Despatie, Garcler le fett, Montréat, Planète rebelle, 2001, $78 \mathrm{p}$.

13. Martin Thibuult, La totalité du paysage, Montréal, Éditions du Noroît, 2001, 72 p.

14. Marc André Brouillette, Vent devant, Montréal, Éditions du Noroît, 2001, 84 p.

15. Maurice Tardif, Autoportraits à la paille creuse, Montréal, Triptyque, 2001, 144 p.

16. Normand de Bellefeuille, Un tisage pour commencer, Québec/Marseille, Écrits des Forges/Éditions Autres temps, 2001, 112 p.

17. Id., La marche de latengle sans son chien. Montréal, Québec Amérique, 1999.

18. Poèmes parus dans Liberté, no 86, 1973.

19. Poème du Chant funèbre pour Ignacto Sanchez Mejias. C'est toutefois le recueil Poète à Neu' York qui a surtout nourri, manifestement, l'écriture de de Bellefeuille. 